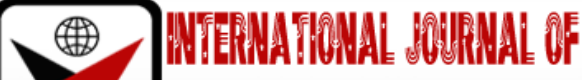

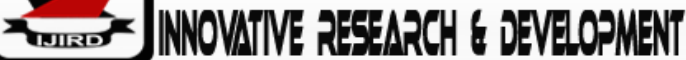

ISSN 2278-0211 (Online)

\section{Influence of COVID-19 Information Graphics Awareness Protocols on the Residents of Ibadan, Nigeria}

Alimi, Adesola Olutoyin
Senior Lecturer, Department of Art, Design and Printing Technology,
The Polytechnic, Ibadan, Nigeria
Olalere Samuel Aderemi
Chief Lecturer, Department of Art, Design and Printing Technology,
The Polytechnic, Ibadan, Nigeria
Adeniji, Julianah Bolade
Senior Lecturer, Department of Art, Design and Printing Technology,
The Polytechnic, Ibadan, Nigeria

\begin{abstract}
:
The heterogeneous nature of the recipients of infographics messages and the interpretation of messages signified in advocacy posters, within recent times, especially in outdoor media, has become a major challenge. This is sometimes attributed to negligence on the part of recipients to the details of signs and symbols and the audience' inability to interpret the codes represented on such advocacy poster due to ignorance, illiteracy or ambiguity in content. This has reduced such signs and symbols to mere decorations in print. The awareness information graphics on the covid19 pandemic came in the form of posters (such as flyers and billboards). Most of these posters contain basic hygiene steps that must be followed by individuals and corporate organizations for the containment of the spread of the virus. However, the history of information graphics in Nigeria is traceable to the practice of outdoor media such as billboard art of the advertising industry. The billboard poster art is a veritable out-of-the-home medium of advertising and publicity with strategic potentials of good use of colours, modelling and creative language. Visual data on Covid-19 awareness protocols can be designed and exposed in the most effective way by bringing graphic images to the front burner while producing such designs for public use at a crucial time of pandemic as this. Relevant literature on information graphics, billboard art and the management protocols of the covid19 pandemic were reviewed. A quantitative research was adopted for descriptive analysis of data from survey with the use of questionnaire while billboard posters were content analysed. Findings revealed that the graphic design elements that form the content of the infographics visuals today may end up as key influencing factors to determine the target audience's choice of information. The paper recommends that various media organizations should endeavour to develop clear, sensible, unambiguous advert visuals with the use of design elements and principles to avoid ambiguity or apathy that may hamper effective communication.
\end{abstract}

Keywords: Awareness protocols, covid19, influence, infographics

\section{Introduction}

Coronavirus disease 2019, otherwise known as COVID-19, is a communicable respiratory disease caused by a new strain of the disease that causes illness in humans. At some point, the virus began in animals. Simultaneously, one or more humans acquired the infection from animals and began transmitting the infection to other humans. The disease began to spread through infected air droplets that are projected during sneezing or coughing. There were doubts and disbeliefs when the first case was reported in Nigeria. The government of Nigeria, after a series of confirmed cases in Ogun and Lagos states as well as in the federal capital territory had to enforce a nationwide lockdown. Meanwhile, The Nigerian Centre for Disease Control (NCDC) came up with awareness regulatory protocols in the form of pictorial data information or information graphics to sensitize the Nigerian public on the need to take precautionary measures in order to curtail the spread of the novel virus. The awareness information graphics came in the form of posters (such as flyers and billboards). Most of these posters contain basic hygiene steps that must be followed by individuals and corporate organizations for the containment of the spread of the virus. However, the history of information graphics in Nigeria is traceable to the practice of outdoor media such as billboard art of the advertising industry.

The billboard poster art is a veritable out-of-the-home medium of advertising and publicity with strategic potentials of good use of colours, modelling and creative language. This form of art interfaces with information graphics such that advocacy messages from government and corporate organizations are exposed to create awareness in the 
interest of the general public. Information graphics is aimed at making communication quick and simple as well as persuasive and interpretive to the targeted or segmented audience (Adesanya, 2001).

Furthermore, information graphics are visual representations rendered in graphics form to present information effectively with clarity and high recall (Alimi, 2009). Information graphics seeks to engage its recipients with the use of relevant visuals to which the recipients are most familiar. This, in turn, will enable interaction between such visual information and the receiving audience. This, according to Agunsoye (1990) will enable a highly receptive audience in a heterogeneous society to respond to visual stimulation of infographics with potentials to persuade the public with strong visual images like outdoor billboard and signages. To this end, visual data on COVID-19 awareness protocols can be designed and exposed in the most effective way by bringing graphic images to the front burner while producing such designs for public use at a crucial time of pandemic as this.

\subsection{Statement of the Problem}

The heterogeneous nature of the recipients of infographics messages and the interpretation of messages signified in advocacy posters, within the recent time, especially in outdoor media has become a major challenge. Adewale, (2011) noted that negligence on the part of recipients to the details of signs and symbols and the audience' inability to interpret the codes represented on such advocacy poster due to ignorance, illiteracy or ambiguity in content (sometimes as a result of printing error) has reduced such signs and symbols to mere decorations in print. Thus, such audience are nonchalant to details of the messages being communicated at a given time. In most cases, some unconventional symbols that are alien to certain recipients of advocacy infographics may not be interpreted to mean what they actually signify. Most recipients tend to gloss over visuals on posters, billboard and transit displays, and pay little or no attention to interpreting the coded signs and symbols contained in such visuals especially the uneducated. Unfortunately, a segment of the public may not have access to most poster advocacy visuals, especially the uneducated that live in rural communities. Hence, the uneducated will depend on others to tell them what to do all of the time. Consequently, this study examines the influence of information graphics in communicating the awareness protocols of the COVID-19 pandemic to the residents of Ibadan.

\section{Literature Review}

Information graphics has been a veritable means of communicating critical information within organizations and communities over the years. Product advertisers in the electronic and outdoor medium have exploited information graphics over time. This essentially has been a strategic expression of messages that are designed with elements of design as well as visuals and typefaces.

Cairo (2013), Lankour et al (2012) and Smiciklas (2012) gave a lucid insight into the capacity of infographics as a critical transmitter of ideas such that when a pictorial data is sent to the brain through the eyes, the information is eventually transformed into a mental image. According to their publications, there is an unequivocal synthesis between what infographics designers do and what visualizers offer to the readers. Their publications further explained that infographics are visual representations of information used in telling stories to increase reader's understanding of specific subjects while the data is presented for the readers to see, read, explore and analyse.

Mackinlay et al (2013), Arntson (2007) and Smiciklas (2012) agreed that information graphics essentially projects visual appeal, simplicity of presentation, clarity of content and conciseness of detail. Their publications explained that infographics must be presented to raise awareness over an issue or an advocacy and share it with the target recipients in the most effective way. Furthermore, information graphics are reflected on web pages to offer a glimpse of details that will be found within the contents of a website. Animated objects, photographs and other basic information are expressed in the form of information graphics.

\section{Theoretical Framework}

This study is predicated on verbal versus visual imaging theory. On the verbal side, the greater emphasis is given to words in the publicity contents in order to evoke the response from the target market. The visual imagery is considered to be more effective than the verbal message. Visual imagery is stored both as pictures and words in brain and this dual processing increases recall level. Also, the visual imagery is considered to be a universal language for the content developers as an image will depict the same thing for audience belonging to different geographical and cultural settings. However, the meaning of a word may be different for different people. In this respect, the visual imagery is very effective. By this theory, textual or written messages will be more effective if reinforced by visual images (Messaris, 1997). In this regard, this theory will assist the study on the influence of infographics awareness posters on the residents of Ibadan and seek to know if the visual protocols on prevention of the spread of the novel corona virus really resonates with the target recipients or whether such pictorial information are just considered to be mere decorations. In a society that is constantly bombarded with digital information, it is considered necessary to determine if such messages are received as effectively as possible and whether intended communication is achieved by its contents.

\section{Methodology}

A combination of survey and content analysis was adopted in this study. The study population was divided into two categories; billboard posters and subjects. The sampling procedure and sample size was discussed in two parts namely:

- Sample for infographics billboards

- Sample for subjects 
A total of ten (10) billboard publicity posters were selected using the purposive sampling technique. The purposively selected titles are one (1) state government advocacy billboard poster, two (2) types of publicity posters from schools, two (2) types of publicity posters from private organizations (NGOs), five (5) internet generated awareness protocol posters on COVID-19 disease. These visuals were selected because of their common reflections of visuals that were quite familiar. A total of 200 people including students, professionals, public servants and market women were selected as sample size for the study using both the stratified and purposive sampling techniques. These respondents were first stratified into two, which are subjects from the academic and non-academic environments. The University of Ibadan and The Polytechnic, Ibadan were purposively selected to represent academic institutions while independent artisans and public servants were selected to represent the non-academic, and non-literate market women to represent the business environment sample in Ibadan. The method adopted for the analysis of data to be collected for this study was based on inferential statistical analysis (e.g., simple percentages).

\section{Findings}

To determine the influence of COVID-19 information graphics awareness protocol posters on the residents of Ibadan. In this paper, a content analysis method was employed with content categories like colour, illustration, typography and visibility to know the extent to which information graphic elements stimulate the interest of the recipients.

\begin{tabular}{|c|c|c|c|c|c|c|c|c|c|}
\hline \multirow{2}{*}{$\begin{array}{c}\text { Infographic } \\
\text { Elements }\end{array}$} & \multirow{2}{*}{ Measurement } & \multicolumn{2}{|c|}{ Internet } & \multicolumn{2}{c|}{ Ngos } & \multicolumn{2}{c|}{ Govt Poster } & \multicolumn{2}{c|}{ Schools } \\
\cline { 2 - 10 } & FREQ. & $\%$ & FREQ. & $\%$ & FREQ. & $\%$ & FREQ. & $\%$ \\
\hline \multirow{4}{*}{ Colour } & Great Extent & 113 & 56.5 & 122 & 61 & 94 & 47 & 118 & 59 \\
\cline { 2 - 10 } & Little Extent & 73 & 36.5 & 56 & 28 & 69 & 34.5 & 52 & 26 \\
\cline { 2 - 10 } & Very Little Extent & 8 & 4 & 13 & 6.5 & 25 & 12.5 & 20 & 10 \\
\cline { 2 - 10 } & No Extent & 6 & 3 & 9 & 4.5 & 12 & 6 & 10 & 5 \\
\hline \multirow{4}{*}{ Typography } & Total & 200 & $(100 \%)$ & 200 & $(100 \%)$ & 200 & $(100 \%)$ & 200 & $(100 \%)$ \\
\hline & Great Extent & 69 & 34.5 & 88 & 44 & 62 & 31 & 78 & 39 \\
\cline { 2 - 10 } & Little Extent & 95 & 47.5 & 81 & 40.5 & 87 & 43.5 & 79 & 39.5 \\
\cline { 2 - 10 } & Very Little Extent & 26 & 13 & 17 & 8.5 & 36 & 18 & 29 & 14.5 \\
\cline { 2 - 9 } & No Extent & 10 & 5 & 14 & 7 & 15 & 7.5 & 14 & 7 \\
\hline & Total & 200 & $(100 \%)$ & 200 & $(100 \%)$ & 200 & $(100 \%)$ & 200 & $(100 \%)$ \\
\hline
\end{tabular}

Table 1: Summary of the Extent to Which Infographics Elements in COVID-19 Awareness Posters Stimulate the Interest of Their Targets

Source: Field Survey (2020)

Findings in table 1 shows that Colour on internet generated visuals on COVID-19 protocol posters stimulate $56.6 \%$ respondent's interest towards compliance, $61 \%$ in NGOs, $47 \%$ in government owned posters, and 59\% on school owned posters. Typography in internet generated visuals on COVID-19 posters stimulate 34.5\% respondent's interest towards the poster, $44 \%$ in NGOs, $31 \%$ in government owned posters. This shows that the use of colour is high in stimulating recipient's interest in NGOs and internet generated visuals on COVID-19 protocol posters while the use of Typography does not stimulate audience interest in all the posters as shown from the data.

\begin{tabular}{|c|c|c|c|c|c|c|c|c|c|}
\hline Design Element & Measurement & \multicolumn{2}{|c|}{ Internet } & \multicolumn{2}{|c|}{ Ngos } & \multicolumn{2}{c|}{ Govt Poster } & \multicolumn{2}{c|}{ Schools } \\
\hline \multirow{4}{*}{ Symbols } & Great Extent & 93 & 46.5 & 96 & 48 & 68 & 34 & 84 & 42 \\
\cline { 2 - 11 } & Little Extent & 78 & 39 & 72 & 36 & 86 & 43 & 69 & 34.5 \\
\cline { 2 - 11 } & Very Little Extent & 18 & 9 & 20 & 10 & 31 & 15.5 & 30 & 15 \\
\cline { 2 - 11 } & No Extent & 11 & 5.5 & 12 & 6 & 15 & 7.5 & 17 & 8.5 \\
\hline \multirow{4}{*}{ Model (Pictures) } & Total & 200 & $(100 \%)$ & 200 & $(100 \%)$ & 200 & $(100 \%)$ & 200 & $(100 \%)$ \\
\hline & Great Extent & 122 & 61 & 105 & 52.5 & 86 & 43 & 101 & 50.5 \\
\cline { 2 - 10 } & Little Extent & 60 & 30 & 72 & 36 & 72 & 36 & 57 & 28.5 \\
\cline { 2 - 10 } & Very Little Extent & 11 & 5.5 & 12 & 6 & 27 & 13.5 & 26 & 13 \\
\cline { 2 - 10 } & No Extent & 7 & 3.5 & 11 & 5.5 & 15 & 7.5 & 16 & 8 \\
\hline \multirow{5}{*}{ Shapes } & Total & 200 & $(100 \%)$ & 200 & $(100 \%)$ & 200 & $(100 \%)$ & 200 & $(100 \%)$ \\
\hline & Great Extent & 72 & 36 & 80 & 40 & 56 & 28 & 66 & 33 \\
\cline { 2 - 10 } & Little Extent & 85 & 42.5 & 71 & 35.5 & 86 & 43 & 80 & 40 \\
\cline { 2 - 9 } & Very Little Extent & 29 & 14.5 & 31 & 15.5 & 39 & 19.5 & 34 & 17 \\
\cline { 2 - 9 } & No Extent & 14 & 7 & 18 & 9 & 19 & 9.5 & 20 & 10 \\
\cline { 2 - 9 } & Total & 200 & $(100 \%)$ & 200 & $(100 \%)$ & 200 & $(100 \%)$ & 200 & $(100 \%)$ \\
\hline
\end{tabular}

Table 2: Summary of the Extent to Which Infographics Elements in COVID-19 Awareness Posters Stimulate the Interest of Their Targets Source: Field Survey (2020)

Findings in table 2 shows that Symbols (COVID precaution visuals) on internet generated visuals on COVID-19 protocol posters stimulate $46.5 \%$ respondent's interest towards the precautionary campaign, 48\% on NGOs, 34\% in 
government owned campaign posters and 42 in school owned posters. Model (Pictures) on internet generated visuals on COVID-19 protocol posters stimulate 61\% respondent's interest towards the campaign, $52.5 \%$ in NGOs $43 \%$ in government owned COVID-19 posters and 50.5\% in school owned visuals. Shapes in internet generated visuals stimulate $36 \%$ respondent's interest towards the campaign, 40\% in government owned, 28\% in NGOs and 33\% in school-based campaigns. This shows that only Model (Pictures) stimulate audience interest on the internet, NGOs, and government owned campaign posters while the use of Symbols and Shapes do not stimulate audience interest in all the campaign posters as shown from the data.

\begin{tabular}{|c|c|c|}
\hline & Frequency & Percentage \\
\hline Very often & 60 & 30.3 \\
\hline Often & 76 & 38.4 \\
\hline Little & 52 & 26.3 \\
\hline Not quite & 10 & 5.1 \\
\hline
\end{tabular}

Table 3: Responses On the Degree of Respondent's Perception of The Visual Images on COVID-19 Infographics Campaign Posters Source: Field Survey (2020)

As indicated in table 3, 76 (38.4\%) respondents pay attention to billboard posters on COVID-19 awareness campaign often. $60(30.3 \%)$ respondents pay attention very often to COVID-19 campaign posters while 52 of the subjects, $(26.3 \%)$ pay little attention to the infographics campaign posters under study. $10(5.1 \%)$ indicated the subject who did not pay attention to the campaign posters under study. This show that majority do not pay attention to billboard posters ofCOVID-19 campaign posters.

\begin{tabular}{|c|c|c|c|c|c|c|c|c|}
\hline $\begin{array}{c}\text { Category of } \\
\text { Visual Images }\end{array}$ & \multicolumn{2}{|c|}{ Internet } & \multicolumn{2}{c|}{ NGOS } & \multicolumn{2}{c|}{ GOVT Poster } & \multicolumn{2}{c|}{ Schools } \\
\hline & Frequency & $\%$ & Frequency & $\%$ & Frequency & $\%$ & Frequency & $\%$ \\
\hline Colour & 113 & 56.5 & 122 & 61 & 94 & 47 & 118 & $59 \%$ \\
\hline Typography & 8 & 4 & 13 & 6.5 & 25 & 12.5 & 20 & 10 \\
\hline Logo & 6 & 3 & 9 & 4.5 & 12 & 6 & 10 & 5 \\
\hline Model & 73 & 36.5 & 56 & 28 & 69 & 34.5 & 52 & 26 \\
\hline
\end{tabular}

Table 4: Responses on the Dominant Visual Images and What They Symbolize

Source: Field Survey (2020)

Table 4 revealed that 113 of the subjects (56.5\%) perceived colours dominant visual on internet campaign posters on COVID-19, 122 (61\%) on NGOs 94(47\%) on government owned campaign visuals and 118(59\%) on school owned information graphics boards as having dominant visual contents. Also, 73 of the subjects (36.5\%) and 69(34.5\%) perceived models on internet and NGOs posters and billboard campaigns as having dominant visuals while $56 \%$ of respondents perceived typography and logo symbols as dominant visual in all the COVID-19 campaign visuals under study. This shows that the use of colour influences the visual imagination of recipients in all the campaign posters under study.

\section{Discussion}

The content analysis however, revealed that colours on internet generated adverts were bright and visible while a high contrast was minimally created to make the design more appealing. It was observed that emphasis was placed on the use of warm and aggressive colours such as red and yellow (bright and visible enough) to attract attention to the product. Large illustration was frequently adopted on NGOs and school posters. The dominance of the model on a poster helps in creating effective awareness for potential target audience. The expressions of logo and house colours were clearly and frequently represented on government posters. The infographics posters reflected legible typefaces on internet generated posters and school-based advocacy posters. Male and female models are frequently portrayed in the graphic rendition of NGO poster visuals. Findings further reveals that recipients of internet, NGOs and school-based posters often pay attention to advocacy posters with the frequency of 76(38.4\%). Finding also shows that $118(59 \%)$ of respondents perceived colours as dominant visual image on internet posters, while 69 (34.5\%) see models on school posters as dominant visual image. To ascertain the preference of culture represented in advocacy posters in this paper, findings revealed that recipients see cultural values projected in NGOs, as relevant and indicated that information that are targeted at them should portray their culture more than the foreign infographic contents.

\section{Conclusion}

Results indicate that internet, NGOs, government-based advocacy posters and school generated visuals under study have graphic design elements that are capable of stimulating the interest of targets. It was revealed that design elements like colour, typography, models and trademarks are perceived to be significant factor in adopting advocacy information graphics that are projected to reflect such elements.

Findings further revealed that symbols and typefaces have the potential to facilitate effective communication especially when such symbols or texts are legible and frequently adopted to communicate to a well-targeted audience. The $38.4 \%$ recipients that pay attention to advocacy posters on COVID-19 through the media sources under study and 56.6\% 
that pay attention to infographics in other media implies that majority of subjects are committed to viewing visuals of their choice of information which has the capacity of influencing their decisions on the choice of such information to which they are mostly attracted.

These findings lend credence to the stand point of Shirley (2016) that humans are most likely influenced by the visual expressions they see in billboards and posters and that they are powerful instruments of the socialization of the youth and educated adults alike as they tend to be attracted significantly to such media that can increase their intellectual capacity. Therefore, the graphic design elements that form the content of the infographics visuals today may likely end up as key influencing factors to determine the choice of information by the target audience, especially, the youth. It follows, then, that, the more certain signs and symbols are portrayed in infographics posters, the more it becomes a platform through which recipients of such information are compelled to make their choices

\section{Recommendations}

In view of the findings of this paper, it is recommended that graphic designers should endeavour to create more compelling advocacy posters that are visually motivating and as well easily interpreted by the target audience. Hence, designers should ensure that communication design elements are maximally employed without being flippant and without any suggestive imagery that may lead to anti-social behaviour. It is also recommended that various media organizations should endeavour to develop clear, sensible, unambiguous advert visuals with the use of design elements and principles to avoid ambiguity or apathy that may hamper effective communication.

The study further recommends that Advertising Practitioners Council of Nigeria (APCON) may need to come up with a more effective standard of vetting advocacy posters as it is done to advertisement billboards to ensure that content is professionally and ethically developed to put the recipients in a good position of benefiting from the information being communicated and possibly advocate for use of local languages more in the designing of such contents despite the media highlighted in this study.

\section{References}

i. Adewale, Moses. 2011. Transit Advertising in Nigeria: An evaluation of Product Advertising on Lagbus in Lagos Metropolis. Unpublished M.A. Thesis Ibadan: University of Ibadan.

ii. Adesanya, Michael. 2001. The influence of advertising on Alcohol consumption among University of Ibadan students. Unpublished B.A. Long Essay. University of Ibadan

iii. Agunsoye, Ayo. 1990. A study of the effects of Television on Adolescents Unpublished M.A. Dissertation, University of Ibadan

iv. Alimi, Adesola Olutoyin. 2009. Basic Principles of Graphics Design and Printing Technology, Ibadan: Crownix Media Concepts.

v. Arntson, Amy. 2007. Graphic Design Basics, Fifth Ed Clark Baxter Wordsworth, Boston: 02210-1202, USA

vi. Cairo, Alberto. 2013. The Functional Art: An introduction to information Graphics and visualization. Beckerly, CA 94710 ISSN 510-524-2178

vii. Krum, Randy. 2013. Cool Infographics: Effective communication with Data visualization and Design, Florida: McgramPrints

viii. Lankour, Jason, Ritchie Josh and Crook Ross 2012. Infographics: The power of visual storytelling. Florida: McgramPrints

ix. Mackinlay, John. Kosara, Rose. and Wallace, Moore. 2013. Data Storytelling: Using visualization to share the human impact of numbers.

x. Messaris, Pam. 1997. Visual Persuasion: The Role of Images in Advertising London: Sage Publications.

xi. Shirley, Mary. 1981. "Influence of Design on Consumer buying decisions". (Journal of Advertising) issue 10, pp 124

xii. Smiciklas, Matt. 2012. The Power of Infographics: Using Pictures to Communicate and Connect with your Audiences, Indianapolis: QUE. 


\section{Appendix}

Plates of Information Graphics on COVID-19 Awareness Protocols

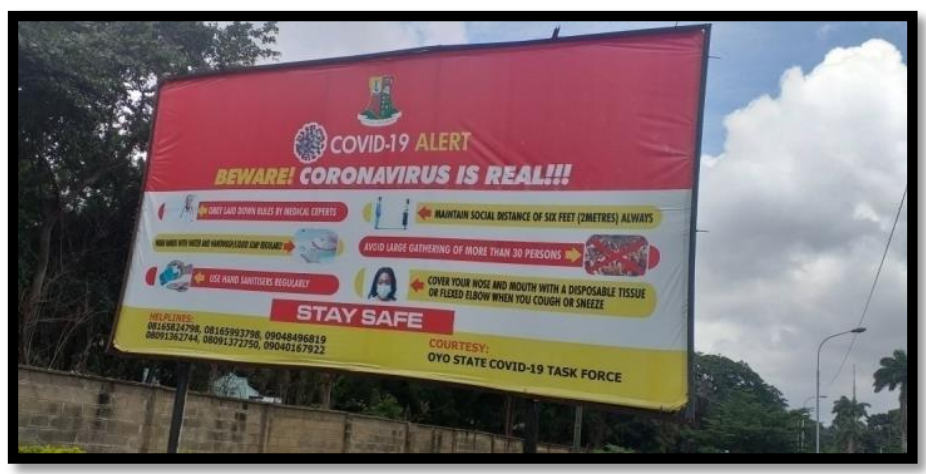

Plate 1: COVID-19 ALERT

Source: Government Owned poster

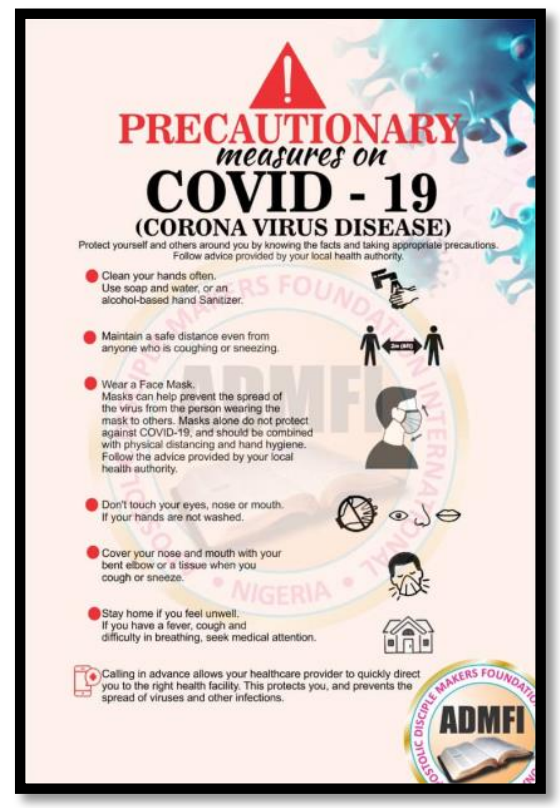

Plate 2: Precautionary Measures on COVID-19 Poster

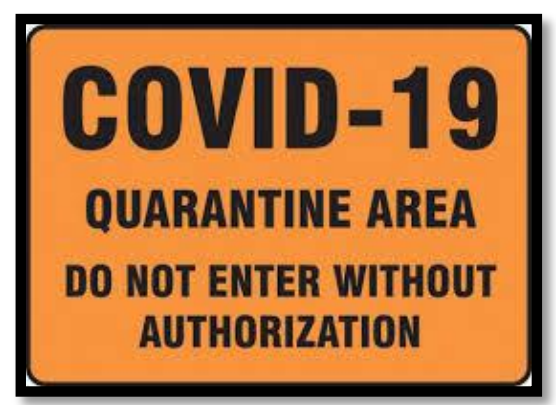

Plate 3: COVID-19

(Internet Generated Poster) 


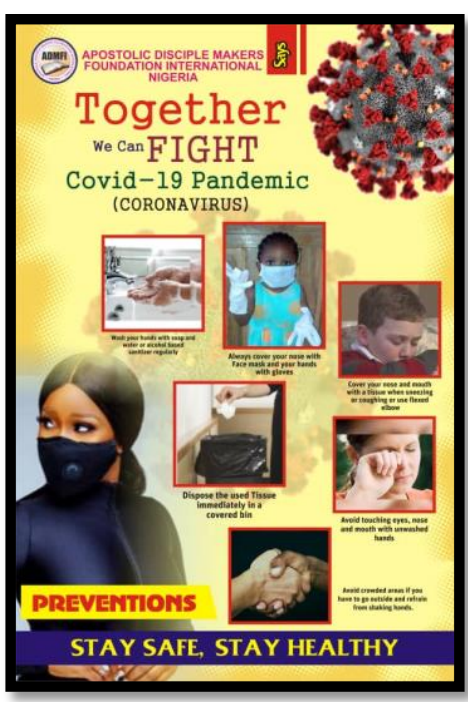

Plate 4: Together We Can Fight COVID-19 Pandemic Poster

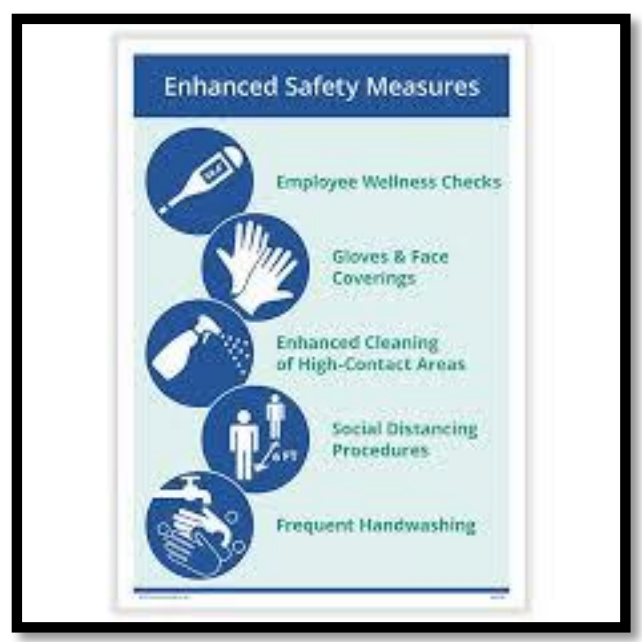

Plate 5: Enhanced Safety Measures

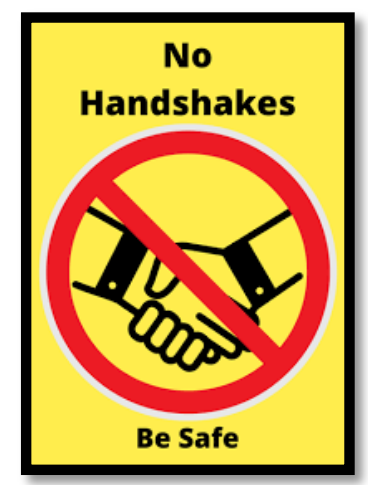

Plate 6: No Handshakes Poster

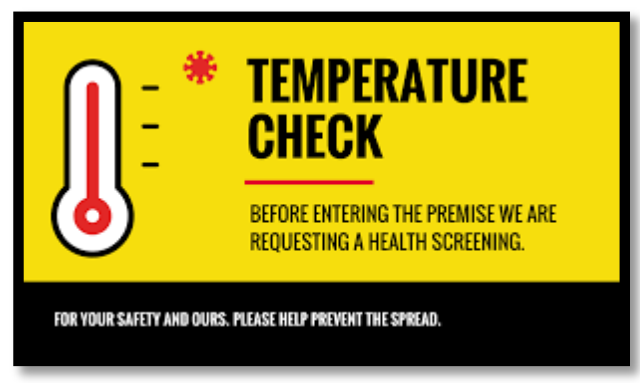

Plate 7: Temperature Check Poster 